\title{
Study of the influence of transducer-electrode and electrode-wall gaps on the acoustic field inside a sonoelectrochemical reactor by FEM simulations
}

\author{
Ignacio Tudela ${ }^{\mathrm{a}}$, Verónica Sáez ${ }^{\mathrm{a}}$, María Deseada Esclapez ${ }^{\mathrm{b}}$, Pedro Bonete ${ }^{\mathrm{b}}$, Hassen Harzalic ${ }^{\mathrm{c}}$, \\ Fabien Baillon ${ }^{c}$, José González-García a , Olivier Louisnard ${ }^{\mathrm{c}, *}$ \\ a Grupo de Nuevos Desarrollos Tecnológicos en Electroquímica: Sonoelectroquímica y Bioelectroquímica, Departamento de Química Física e Instituto Universitario de Electroquímica, \\ Universidad de Alicante, Ap. Correos 99, 03080 Alicante, Spain \\ ${ }^{\mathrm{b}}$ Grupo de Fotoquímica y Electroquímica de Semiconductores, Departamento de Química Física e Instituto Universitario de Electroquímica, Universidad de Alicante, Ap. Correos 99, \\ 03080 Alicante, Spain \\ c Ecole des Mines Albi, Centre RAPSODEE, Université de Toulouse, FRE CNRS 3213, 81013 Albi, France
}

\section{A B S T R A C T}

The influence of transducer-electrode and electrode-wall gaps on the spatial distribution of the acoustic pressure inside a sonoelectrochemical reactor has been studied by employing a linear acoustics-based model accounting for vibrations of the reactor walls. A FEM (Finite Elements Method) software package was used in order to simulate the response curves of the system, the distribution of the acoustic pressure and the deformation of the surrounding walls. Attenuation of the acoustic energy in the liquid due to the presence of cavitating bubbles was introduced using an attenuation coefficient, enabling to study its influence in the available working frequency range of the ultrasonic transducer (19-21 kHz). Acoustic energy stored in the liquid was plotted as a function of frequency in order to obtain the response curves of the sonoelectrochemical system, exhibiting different resonance peaks for each studied configuration. The proper election of the reactor configuration and setting of the working frequency of the transducer allows the formation of pressure antinodes near the electrode favouring cavitation events in this zone, and helping to minimize the cavitation-induced erosion of the sonotrode commonly observed during experiments.

Keywords:

Acoustic pressure

Cavitation

Finite elements method

Resonance

Sonoelectrochemistry

Ultrasound

\section{Introduction}

The synergic combination of electrochemical processes with ultrasound has been an active research in recent years due to its potential use in many different applications [1]. Many studies are found in the literature referred to its use in environmental remediation as part of hybrid technologies for the degradation of organic pollutants in aqueous media [2-4], but also referred to its use as a non-chemical treatment [5] and as its most common application, direct sonoelectrolysis [6].

Sonoelectrochemical processes have been deeply studied by the authors in two different application areas: the sonoelectrodeposition of $\mathrm{PbO}_{2}$ on various substrates [7-9] and the sonoelectrochemical removal of organic compounds such as trichloroacetic acid [10] or perchloroethylene [11] in water. The latter constitutes an interesting option for environmental remediation, as complete degradation of $\mathrm{C}-\mathrm{Cl}$ bonds, which are toxic in nature, is achieved [12]. However, the high energy consump-

\footnotetext{
* Corresponding author. Tel.: +330563493062.

E-mail address: louisnar@enstimac.fr (O. Louisnard).
}

tion associated to the application of ultrasound within the reactor and the non-homogeneous distribution of cavitation makes current sonoelectrochemical water treatment industrially unfeasible from an economic point of view. In order to improve the sonication efficiency related to the energy consumption, the optimization of all kinds of sonoreactors must be faced taking into account building materials and geometric design, and also setting an optimum working frequency of the overall sonoelectrochemical system [13]. Therefore, a correct understanding of the acoustic field structure in the reactor is needed, prior to its optimization and scale-up. This consideration would allow us to predict the cavitating zones inside the reactor in order to effectively design large scale reactors [14] and to control the extent of transient and stable cavitation events [15].

In the last 15 years, quite a few research groups have tried to model the spatial distribution of the acoustic pressure inside a sonoreactor by numerical methods. Dähnke and Keil developed a 3D model to calculate linear pressure fields in liquids with an inhomogeneous distribution of cavitation bubbles [16]. They compared the results with experimental data, obtaining a good agreement but observing some deviations [17]. Liu et al. [18] used Finite Element Methods (FEM) to simulate coupled vibration modes in an 
ultrasonic cleaning tub in order to study the effect of the presence of a washing object. They concluded that the acoustic modes depended on the object location, its mechanical restraint conditions and its physical properties (Young modulus and density). More recently, Yasui et al. [19] simulated a rectangular sonoreactor accounting for vibrations of the vessel wall. They showed the effect of the wall thickness for two frequencies (100 and $140 \mathrm{kHz}$ ), employing various attenuation coefficients in order to observe the effect of cavitating bubbles on the acoustic field.

Numerical simulations have also been evaluated by the authors in recent years. An initial approach to the calculation of the acoustic field by FEM techniques was made in order to mechanically characterize a commercial $20 \mathrm{kHz}$ sonoreactor [20], where the liquid boundary in contact with air was treated as infinitely soft and the vessel boundaries were treated as infinitely rigid walls. Further studies, where a first attempt to optimize a sonoreactor was carried out [21], focused on the geometry of the reactor (cell dimensions, liquid volume and ultrasonic horn position), treating the cell boundaries as infinitely soft walls. FEM simulations and experimental observations verified that the fast decrease in intensity when increasing distance from the horn could be reversed to an increase due to multiple reflections supported. And more recently, an analysis of the response of an experimental sonoreactor equipped with a cooling jacket excited by a cup-horn transducer was performed [22]. Simulations were carried out accounting for the vibration of the reactor walls, pointing out the transmission of sound into the cooling jacket. Ultrasound frequency was systematically varied in the range available for the generator, enabling to seek the resonance frequencies of the system, which were characterized by global parameters as functions of frequency.

In this work, we analyzed the effect which may present the distance between the transducer emitter surface and the electrode and the distance between the electrode and the bottom reactor wall in a cylindrical sonoelectrochemical reactor designed by CAD tools. The main goal was to qualitatively estimate the zones where cavitation could take place near resonance frequencies, taking into account two different physical phenomena: the application of ultrasound in the working liquid and the deformation of the reactor walls. For this purpose, the previously developed model accounting for vibrations of the boundaries [22] was employed to estimate the acoustic field inside a sonoelectrochemical reactor.

\section{The simulated system}

The cylindrical reactor simulated is shown in Fig. 1. The reactor is built on polycarbonate (PC) in order to visualize the cavitation fields inside the reactor in further experimental work with luminol. The flange which holds the reactor to the transducer is made of polypropylene (PP) and titanium (Ti) has been considered for the electrode. The horn tip is based on current $20 \mathrm{kHz}$ UIP1000hd transducer [23] provided by Hielscher Ultrasonics $\mathrm{GmbH}$.

\section{FEM simulations}

\subsection{Theoretical model}

As mentioned before, the acoustic field and the deformation of the reactor walls were calculated by following the simulation strategies exposed in previous works by the authors. Therefore, a short recapitulation of the key equations previously developed is included in the following sections. For deeper discussion on this topic, refer to Ref. [22].

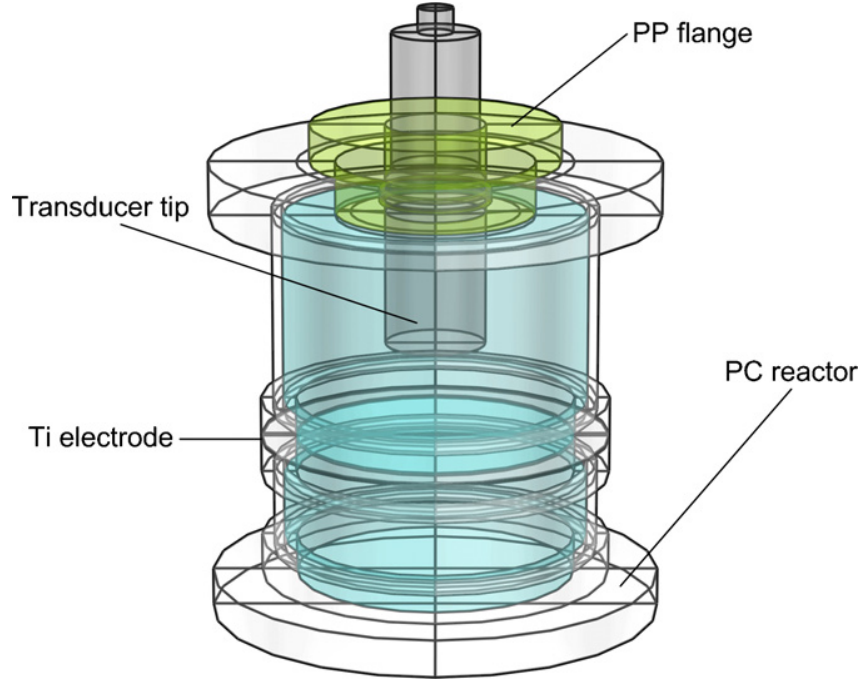

Fig. 1. PC cylindrical electrochemical reactor designed for this study.

\subsubsection{Linear acoustics in the working liquid}

In order to calculate the propagation of sound waves through the liquid the next expression, known as the Helmholtz equation, was considered:

$\nabla^{2} P+\left(\frac{\omega}{c_{l}}\right)^{2} P=0$

where $P$ is the acoustic pressure, $\omega$ is the angular frequency and $c_{1}$ is the sound speed of the liquid.

This equation must be supplemented with adequate boundary conditions. First, assuming a horn radiating surface normal displacement of amplitude $U_{0}$, the boundary condition is obtain by imposing the inward acceleration of the liquid as:

$\left(\frac{1}{\rho_{\mathrm{l}}} \nabla P\right) \cdot \mathbf{n}=\omega^{2} U_{0}$

where $\rho_{1}$ is the density of the liquid. The horn lateral wall is considered as an infinitely rigid boundary, so that the inward liquid acceleration on this boundary is set to zero:

$\left(\frac{1}{\rho_{1}} \nabla P\right) \cdot \mathbf{n}=0$

Finally, for all interfaces between the liquid and the deformed solid domain (either electrode or the reactor walls), the liquid acceleration must match the solid one, and the boundary condition reads:

$\nabla P \cdot \mathbf{n}=\rho_{1} \omega^{2} \mathbf{U}_{\mathrm{s}} \cdot \mathbf{n}$

where $\mathbf{U}_{\mathrm{s}}$ is the complex amplitude of the displacement field of the solid, which coupled calculation is described in the next section. In those expressions, $\mathbf{n}$ is the normal pointing outward the liquid.

\subsubsection{Vibrations of the solids}

The vibrations of the reactor walls are calculated as in Ref. [22], using the elasto-dynamic theory, assuming elastic deformation for all materials, neglecting volume forces and assuming monoharmonic vibrations:

$-\rho_{s} \omega^{2} \mathbf{U}_{\mathrm{s}}=\nabla \cdot \overline{\bar{\Sigma}}$

where

$\overline{\bar{\Sigma}}=\frac{E v}{(1-2 v)(1+v)}(\operatorname{Tr} \overline{\overline{\boldsymbol{\varepsilon}}}) \overline{\overline{\mathbf{I}}}+\frac{E}{1+v} \overline{\overline{\boldsymbol{\varepsilon}}}$

$\overline{\overline{\boldsymbol{\varepsilon}}}=\frac{1}{2}\left[\overline{\bar{\nabla}} \mathbf{U}_{\mathrm{s}}+{ }^{\mathbf{T}} \stackrel{\bar{\nabla}}{\nabla} \mathbf{U}_{\mathrm{s}}\right]$ 
where $\mathbf{U}_{\mathrm{s}}$ is the complex displacement field in the solid, $\overline{\overline{\boldsymbol{\Sigma}}}$ and $\overline{\overline{\boldsymbol{\varepsilon}}}$ are the elastic stress tensor and strain tensor, respectively, $\rho_{\mathrm{s}}$ is the solid density, $v$ is the Poisson ratio of the solid, $\overline{\bar{I}}$ is the identity tensor, $T r$ is the trace operator and $E$ is the Young modulus.

Eq. (5) must be supplemented with the following boundary conditions. The solid boundaries in contact with air are assumed free, so that the three components of the stress $\overline{\bar{\Sigma}} \cdot \boldsymbol{n}$ are ascribed to 0 on such surfaces.

Finally on the interfaces between the liquid and the solid, the stress imposed on the solid is just the normal pressure stress exerted by the liquid, and the condition reads:

$\overline{\bar{\Sigma}} \cdot \mathbf{n}=-P \cdot \mathbf{n}$

where $P$ is the acoustic pressure field in the liquid evaluated on the interface. Again, $\mathbf{n}$ is the normal pointing outward the liquid.

It must be noted that Eqs. (5) and (8) are vectorial. The set of Eqs. (4) and (8) constitute the correct acoustic-structure interface conditions.

\subsubsection{Energy dissipation}

In order to account for the energy dissipation by cavitating bubbles, the next energy conservation expression was employed, assuming mono-harmonic waves in steady state:

$\underbrace{\iint-\frac{1}{2} \Re\left(P \mathbf{V}^{*}\right) \cdot \mathbf{n} d S}_{P_{\text {active }}}=\underbrace{\iint-\frac{1}{2} \Re\left(P \mathbf{V}^{*}\right) \cdot \mathbf{n} d S}_{P_{\text {bound }}}+\underbrace{\iiint\left\langle\boldsymbol{\Pi}_{\text {diss }}\right\rangle d V}_{P_{\text {diss }}}$

where $\Re$ denotes the real part of a complex number, $V$ is the liquid volume, $S$ is the surface (for both sonotrode and solid boundaries) and $\mathbf{V}^{*}$ denotes the complex amplitude of the acoustic velocity field.

The first and second terms are respectively the power entering the liquid through the sonotrode radiating surface $\left(P_{\text {active }}\right)$ and the power lost through the rest of the liquid boundaries $\left(P_{\text {bound }}\right)$. When considering either infinitely soft or rigid boundaries, the second term vanishes $\left(P_{\text {bound }}=0\right)$. In our more complex case where $S_{\text {boundaries }}$ was the internal wall of the PC reactor, the PP flange and the Ti surface in contact with the liquid, almost the whole external surface of the reactor was in contact with air, and therefore, not being constrained. $P_{\text {bound }}$ is still zero for a perfectly elastic vessel formed of external, non-dissipative, unconstrained elastic solid boundaries which do not transmit any wave to the external air. Therefore, we also assumed that $P_{\text {bound }}=0$ in this study. On the other hand, the third term in Eq. (9) represents the power dissipated in the medium $\left(P_{\text {diss }}\right)$, where an arbitrary, spatially uniform attenuation coefficient, $\alpha$, was introduced as follows:

$\left\langle\boldsymbol{\Pi}_{\text {diss }}\right\rangle=\alpha \frac{|P|^{2}}{\rho_{l} C_{l}}$

\subsubsection{Obtaining of response curves}

Among the different scalar quantities representative of the global vibrations of the system (i.e. average acoustic pressure in the liquid, average acoustic energy stored in the solid walls, etc.), we chose to plot the average acoustic energy stored in the liquid domains $\left(E_{a}\right)$ as a function of frequency, according to the following equation:

$$
E_{a}=\iiint_{V}\left(\frac{1}{4} \rho_{l}|\mathbf{V}|^{2}+\frac{1}{4} \frac{|P|^{2}}{\rho_{l} c_{l}^{2}}\right) d V
$$

where $\mathbf{V}$ denotes the real amplitude of the acoustic velocity field.

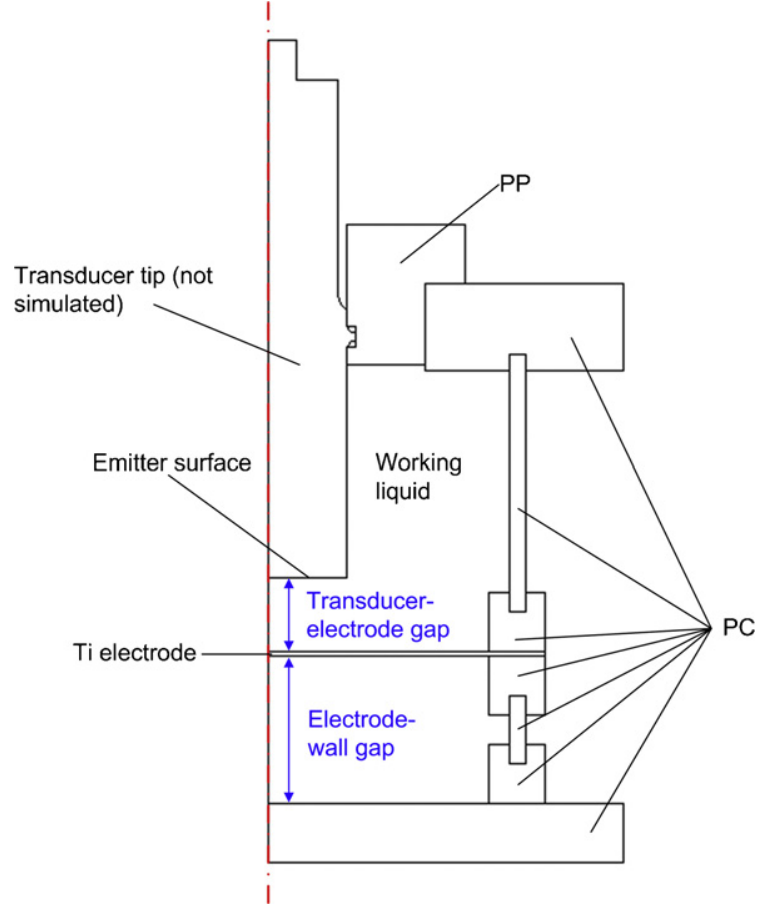

Fig. 2. Simulated geometry. Only one-half of the reactor is drawn. Modified distances are highlighted in blue. (For interpretation of the references to color in text, the reader is referred to the web version of the article.)

\subsection{Resolution procedures}

The simulated set-up is represented in Fig. 2, where only one half of a cut plane is shown. The horn tip $(d=40 \mathrm{~mm})$ emitted in the liquid, limited by the PP flange and PC walls of different thickness $(4-20 \mathrm{~mm})$. The liquid split in two different domains as the Ti electrode ( $1 \mathrm{~mm}$ thick) was placed in different heights. The liquid was water with properties $\rho_{\mathrm{l}}=1000 \mathrm{~kg} \mathrm{~m}^{-3}$ and $c_{\mathrm{l}}=1500 \mathrm{~m} \mathrm{~s}^{-1}$. The elastic properties were $E=6.35 \mathrm{GPa}, v=0.48$ and $\rho_{\mathrm{s}}=898 \mathrm{~kg} \mathrm{~m}^{-3}$ for the PP flange; $E=2.38 \mathrm{GPa}, v=0.36$ and $\rho_{\mathrm{S}}=1200 \mathrm{~kg} \mathrm{~m}^{-3}$ for the PC walls and $E=102.7 \mathrm{GPa}, v=0.34$ and $\rho_{\mathrm{S}}=4507 \mathrm{~kg} \mathrm{~m}^{-3}$ for the Ti electrode. The sonotrode had a working frequency range from 19 to $21 \mathrm{kHz}$, assuming that it would automatically adjust to an optimum frequency where the electric impedance was minimal, with the main oscillation developed along with axis-direction. The emitter surface of the transducer was assumed to vertically vibrate with an amplitude of $25 \times 10^{-6} \mathrm{~m}$.

Three different configurations were studied (Table 1), which were named referring to the emitter-electrode and electrode-wall gaps as a factor of the wave-length $(\lambda=7.5 \mathrm{~cm})$ for water when the frequency is $20 \mathrm{kHz}$, the speed of sound is $1500 \mathrm{~m} \mathrm{~s}^{-1}$ and the density is $1000 \mathrm{~kg} \mathrm{~m}^{-3}$.

Eqs. (1), (5) and (9) were numerically solved with COMSOL Multiphysics package, where both linear acoustics and structural mechanics application modes were employed in order to obtain the spatial distribution of the acoustic pressure in the working liquid and the deformation of the reactor walls. Due to the complexity

Table 1

Distances between the transducer emitter surface and the electrode and between the electrode and the bottom wall of the reactor for each studied configuration, $\lambda=7.5 \mathrm{~cm}\left(20 \mathrm{kHz}, 1500 \mathrm{~m} \mathrm{~s}^{-1}, 1000 \mathrm{~kg} \mathrm{~m}^{-3}\right)$.

\begin{tabular}{lll}
\hline Configuration & Transducer-electrode gap & Electrode-wall gap \\
\hline$\lambda / 4-\lambda$ & $\lambda / 4$ & $\lambda$ \\
$\lambda / 2-3 \lambda / 4$ & $\lambda / 2$ & $3 \lambda / 4$ \\
$3 \lambda / 4-\lambda / 2$ & $3 \lambda / 4$ & $\lambda / 2$ \\
\hline
\end{tabular}




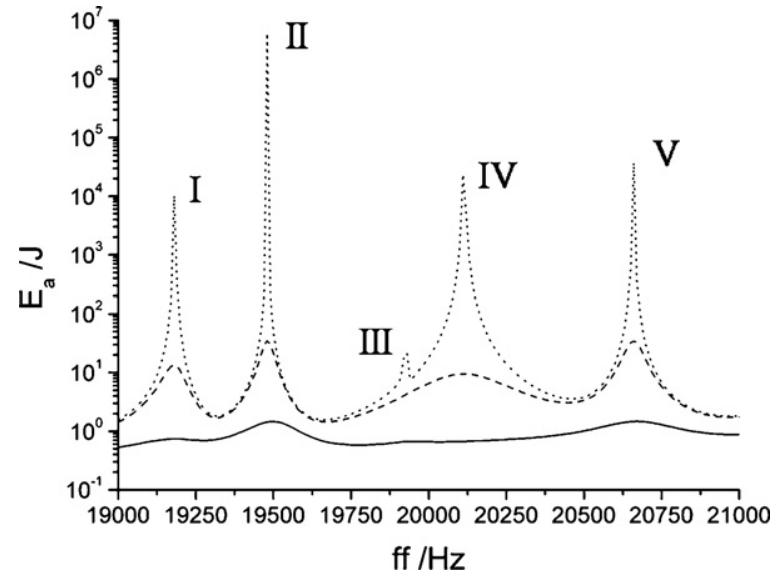

Fig. 3. Average acoustic energy stored in liquid in configuration $\lambda / 4-\lambda$ for various attenuation coefficients: $\alpha=0 \mathrm{~m}^{-1}$ (dotted line), $\alpha=1 \mathrm{~m}^{-1}$ (dashed line) and $\alpha=5 \mathrm{~m}^{-1}$ (solid line).

of the geometry, the number of calculations needed and the thin mesh required (the mesh consisted of more than $10^{5}$ triangular elements in each studied configuration, with an emphasis on meshing the liquid-solid interface and the Ti electrode domain), we chose the axial 2D mode due to the need of extremely high computer resources in order to carry out the study in the 3D mode. This bidimensional mode enables to obtain much finer meshes, although three-dimensional, non symmetrical modes of the vibrating solid domains (i.e. PC reactor, PP flange and Ti electrode) are not calculated. Nevertheless, non symmetrical modes have no chance to be excited for a purely symmetric motion of the transducer in the frame of linear acoustics. The method used here does not actually compute the eigenmodes of the system, but its response to a harmonic driving at variable frequency. Therefore, simulations with the 3D mode would have yielded the same results for the symmetric configuration studied. The UMFPACK [24] solver in parametric mode was employed to obtain the response curves of the system in the working frequency range of the transducer. For this purpose, the frequency was varied by small steps $(10 \mathrm{~Hz})$ in the working range of the transducer $(19-21 \mathrm{kHz})$.

\section{Results}

\subsection{Configuration $\lambda / 4-\lambda$}

For the first studied configuration, the response curves shown in Fig. 3 (average acoustic energy stored in the liquid) were obtained for different attenuation coefficients $\left(\alpha=0,1\right.$ and $\left.5 \mathrm{~m}^{-1}\right)$ in the working frequency range of the transducer. Five resonance peaks were observed when no energy dissipation was considered, which varied or even disappeared when increasing attenuation (Table 2). Those peaks corresponded to global resonances of the whole mechanical system formed by the liquid coupled with the PP flange, the PC reactor and the Ti electrode walls. The amplitude of these

Table 2

Evolution of the resonance frequencies for configuration $\lambda / 4-\lambda$ at various attenuation factors.

\begin{tabular}{llll}
\hline Resonances & \multicolumn{2}{l}{$\alpha\left(\mathrm{m}^{-1}\right)$} & 5 \\
\cline { 2 - 4 } & 0 & 1 & 19,180 \\
\hline Peak I (Hz) & 19,180 & 19,180 & 19,500 \\
Peak II (Hz) & 19,480 & 19,480 & 19,970 \\
Peak III (Hz) & 19,930 & - & - \\
Peak IV (Hz) & 20,110 & 20,110 & 20,670 \\
Peak V (Hz) & 20,660 & 20,660 & \\
\hline
\end{tabular}

Table 3

Evolution of the resonance frequencies for configuration $\lambda / 2-3 \lambda / 4$ at various attenuation factors.

\begin{tabular}{llll}
\hline Resonances & \multicolumn{2}{l}{$\alpha\left(\mathrm{m}^{-1}\right)$} & 5 \\
\cline { 2 - 4 } & 0 & 1 & 19,460 \\
\hline Peak I (Hz) & 19,420 & 19,420 & - \\
Peak II (Hz) & 20,070 & 20,080 & - \\
Peak III (Hz) & 20,190 & 20,190 & 20,420 \\
Peak IV (Hz) & 20,400 & 20,400 & \\
\hline
\end{tabular}

global resonance peaks should be infinite when no attenuation is considered, which means that an infinite accumulation of acoustic energy would take place in the whole sonoelectrochemical system. Thus, the finite maxima of the peaks appearing hereinafter for $\alpha=0 \mathrm{~m}^{-1}$ are only due to the discretization of the frequency axis.

For $\alpha=5 \mathrm{~m}^{-1}$, peaks II and V presented a higher acoustic energy stored in the liquid by far. Therefore, the spatial distribution of the peak acoustic pressure was analyzed in both cases and is shown in Figs. 4 and 5, where dark red regions represent main pressure antinodes and dark blue regions represent main nodes, and the grey line displays the magnified deformed boundary in order to illustrate the coupling between the vibrations of the solid walls and the fluid. For peak II attenuation does not play a great influence in the distribution of the acoustic field, as just a few non-important pressure nodes are affected. However, the spatial distribution of the acoustic pressure at peak $\mathrm{V}$ is clearly affected, as a pressure antinode becomes more evident on the emitter surface, and part of the structure of the acoustic field is modified. Still, the main antinode remains on the electrode surface opposite to the reactor bottom wall. Coupling of the deformation of the Ti electrode and the formation of alternated pressure antinodes on both surfaces of the electrode is clearly seen for all the studied attenuation coefficients at peak V. Also, a low-profile antinode stays on the bottom wall surface in the latter case. All these observed features demonstrate that attenuation not only affects the amplitude of the acoustic pressure (see figure captions), but also the structure of the acoustic field.

\subsection{Configuration $\lambda / 2-3 \lambda / 4$}

For the second studied configuration, four resonance peaks were observed (Fig. 6). Peaks II and III disappeared at the highest attenuation factor, while frequency slightly changed for the remaining peaks (Table 3 ).

If we check the spatial distribution of the peak acoustic pressure for peak I (Fig. 7), we observe that the structure of the acoustic field was slightly modified, with the most noticeable change occurring near the emitter surface (the main antinode came closer to the transducer). However, attenuation strongly affected the structure of the acoustic field for peak IV (Fig. 8), as an antinode on the emitter surface grew in both size and relative pressure amplitude to become the second most important antinode from the peak acoustic pressure point of view. Distribution of nodes and antinodes between the electrode and the wall was completely modified, and as we noted before for peak $\mathrm{V}$ in the first configuration, the main antinode remained on the electrode surface. Again, coupling of the vibration of the electrode and the appearance of pressure antinodes on the electrode surfaces is observed, especially at peak $\mathrm{V}$.

\subsection{Configuration $3 \lambda / 4-\lambda / 2$}

For the last studied configuration, six resonance peaks were observed (Fig. 9). Peaks I, and III disappeared with the highest attenuation factor, while frequency changed for peaks II and V (Table 4). 


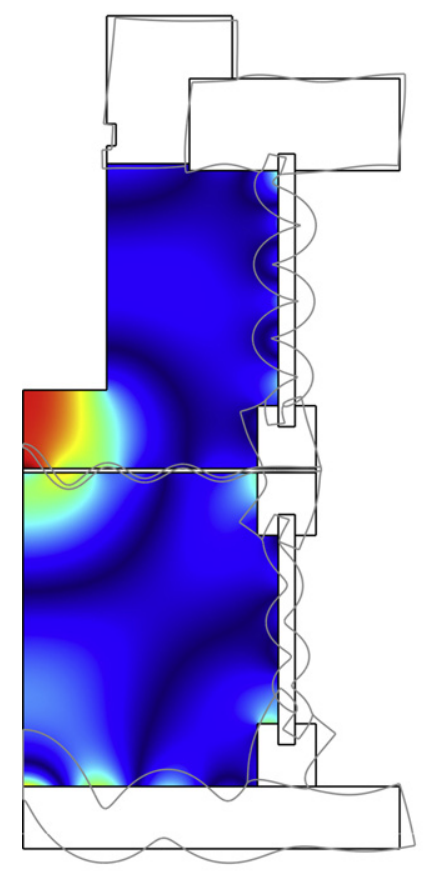

$\alpha=0 \mathrm{~m}^{-1}$

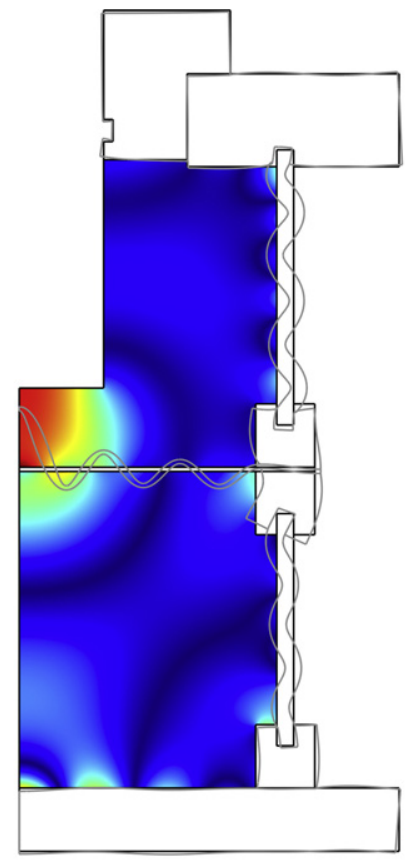

$\alpha=1 \mathrm{~m}^{-1}$

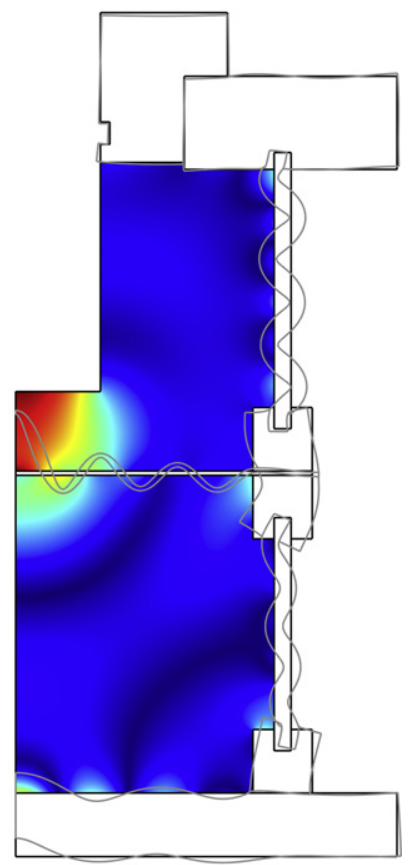

$\alpha=5 \mathrm{~m}^{-1}$

Fig. 4. Spatial distribution of the peak acoustic pressure $|P|$ in the reactor working in configuration $\lambda / 4-\lambda$ near resonance peak II for increasing attenuation coefficients. Dark blue regions represent pressure nodes while dark red regions correspond to pressure antinodes. The color-scales are different for each image. $|P|$ max $=1.96 \times 10^{5}$ bar $\left(\alpha=0 \mathrm{~m}^{-1}\right), 4.76 \times 10^{2} \operatorname{bar}\left(\alpha=1 \mathrm{~m}^{-1}\right)$ and $1.01 \times 10^{2} \operatorname{bar}\left(\alpha=5 \mathrm{~m}^{-1}\right)$. Deformation of the solid boundaries at $\omega t=n \times 2 \pi$ is magnified. (For interpretation of the references to color in text, the reader is referred to the web version of the article.)

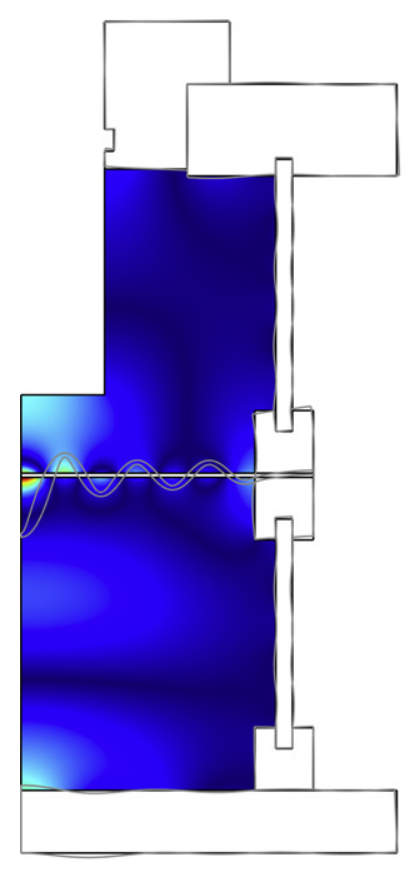

$\alpha=0 \mathrm{~m}^{-1}$

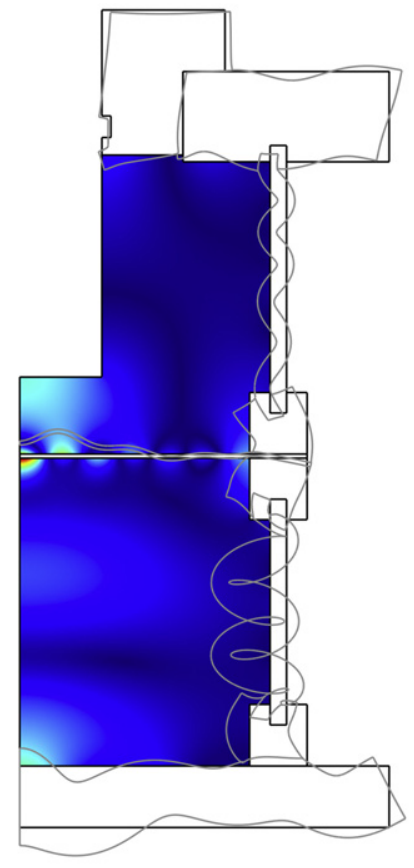

$\alpha=1 \mathrm{~m}^{-1}$

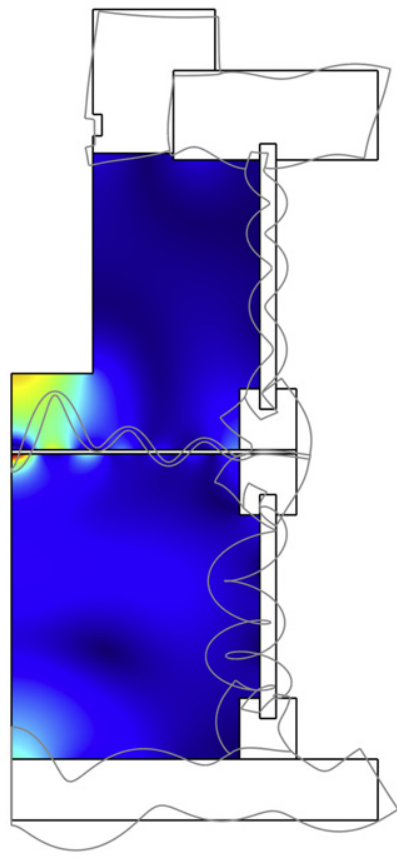

$\alpha=5 \mathrm{~m}^{-1}$

Fig. 5. Spatial distribution of the peak acoustic pressure $|P|$ in the reactor working in configuration $\lambda / 4-\lambda$ near resonance peak $V$ for increasing attenuation coefficients. Dark blue regions represent pressure nodes while dark red regions correspond to pressure antinodes. The color-scales are different for each image. $|P|$ max $=2.67 \times 10^{4}$ bar $\left(\alpha=0 \mathrm{~m}^{-1}\right), 8.18 \times 10^{2} \operatorname{bar}\left(\alpha=1 \mathrm{~m}^{-1}\right)$ and $1.67 \times 10^{2}$ bar $\left(\alpha=5 \mathrm{~m}^{-1}\right)$. Deformation of the solid boundaries at $\omega t=n \times 2 \pi$ is magnified. (For interpretation of the references to color in text, the reader is referred to the web version of the article.) 


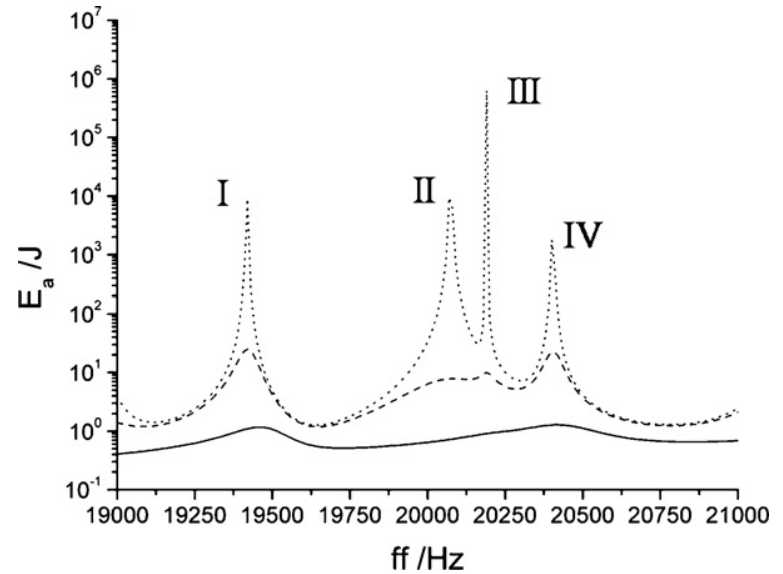

Fig. 6. Average acoustic energy stored in liquid in configuration $\lambda / 2-3 \lambda / 4$ for various attenuation coefficients: $\alpha=0 \mathrm{~m}^{-1}$ (dotted line), $\alpha=1 \mathrm{~m}^{-1}$ (dashed line) and $\alpha=5 \mathrm{~m}^{-1}$ (solid line).

The features of the acoustic field for peak II (Fig. 10) were very similar to those observed for the lowest resonance peak of the previously studied configurations: a main antinode on the emitter surface and few changes on the structure of the acoustic field when attenuation is increased. The same thing occurred for peak VI (Fig. 12) related to the highest resonance peaks from previous configurations: a pressure antinode became more evident on the emitter surface when attenuation increased, but the main antinode remained on the electrode surface. Again, a low-profile antinode formed at the bottom wall of the reactor.

But if we take a look to peak V (Fig. 11), we notice that the acoustic field stayed aside from the other peaks previously commented. When no attenuation was considered, a great, low pressure antinode stayed on the wall surface, while small, high pressure
Table 4

Evolution of the resonance frequencies for configuration $3 \lambda / 4-\lambda / 2$ at various attenuation factors.

\begin{tabular}{llll}
\hline Resonances & \multicolumn{2}{l}{$\alpha\left(\mathrm{m}^{-1}\right)$} & 5 \\
\cline { 2 - 4 } & 0 & 1 & - \\
\hline Peak I (Hz) & 19,250 & 19,250 & 19,460 \\
Peak II (Hz) & 19,430 & 19,430 & - \\
Peak III (Hz) & 19,750 & 19,750 & - \\
Peak IV (Hz) & 20,040 & 20,040 & 20,600 \\
Peak V (Hz) & 20,580 & 20,580 & 20,930 \\
Peak VI (Hz) & 20,930 & 20,930 & \\
\hline
\end{tabular}

antinodes alternated in both sides of the electrode. No significant antinodes were observed in the upper liquid domain, a very interesting feature in order to avoid cavitation-induced erosion of the sonotrode emitter surface. This situation changed when attenuation was considered $\left(\alpha=1 \mathrm{~m}^{-1}\right)$, as the great antinodes between the electrode and the reactor wall were almost gone while new antinodes formed between the emitter surface and the electrode. For the highest attenuation factor, the antinode near the emitter became more evident, but its peak acoustic pressure remained significantly lower than the pressure amplitude near the electrode. Finally, due to the greater distance between the emitter and the electrode in this configuration, it is very easy to see how the deformation of the electrodic domain and the appearance of alternated pressure antinodes and nodes near both surfaces of the electrode are perfectly coupled in all the peaks shown in Figs. 10-12.

\section{Discussion}

In order to check which configuration would show a better performance, we compared the response curves for $\alpha=5 \mathrm{~m}^{-1}$ (Fig. 13). Higher values were reached for configuration $\lambda / 4-\lambda$, where peaks II and $V$ were clearly seen. Configuration $\lambda / 2-3 \lambda / 4$ could also be inter-

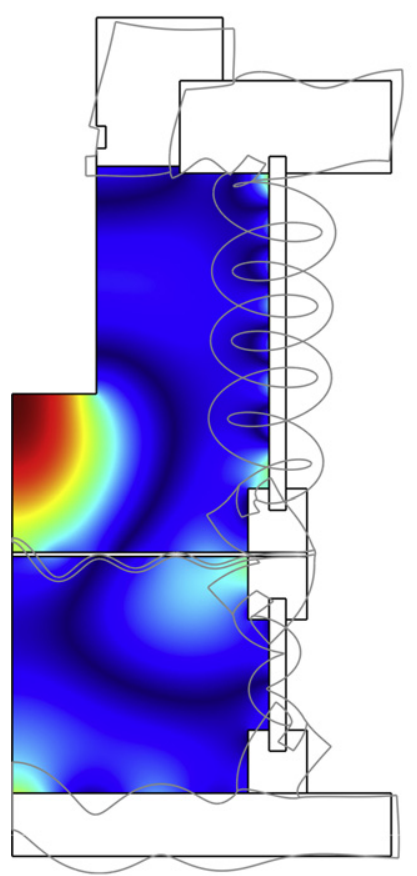

$$
\alpha=0 \mathrm{~m}^{-1}
$$

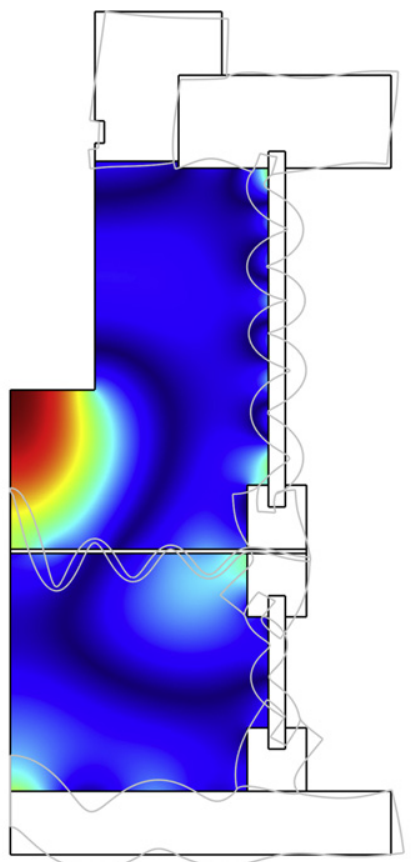

$\alpha=1 \mathrm{~m}^{-1}$

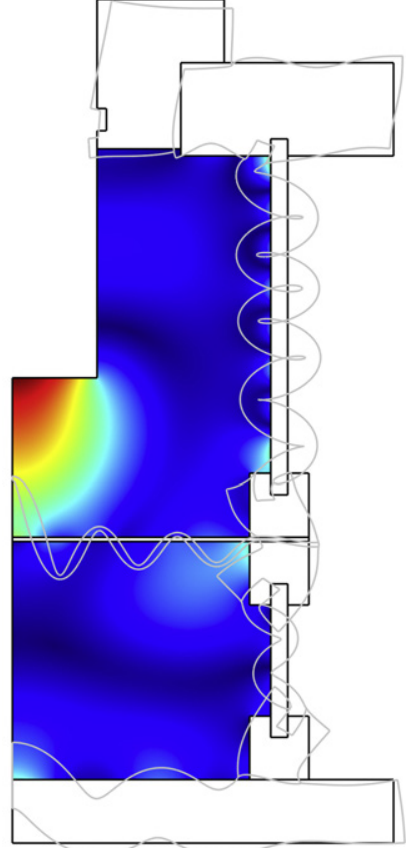

$\alpha=5 \mathrm{~m}^{-1}$

Fig. 7. Spatial distribution of the peak acoustic pressure $|P|$ in the reactor working in configuration $\lambda / 2-3 \lambda / 4$ near resonance peak I for increasing attenuation coefficients. Dark blue regions represent pressure nodes while dark red regions correspond to pressure antinodes. The color-scales are different for each image. $|P| \mathrm{max}=6.50 \times 10^{3}$ bar $\left(\alpha=0 \mathrm{~m}^{-1}\right), 3.49 \times 10^{2} \operatorname{bar}\left(\alpha=1 \mathrm{~m}^{-1}\right)$ and $8.15 \times 10$ bar $\left(\alpha=5 \mathrm{~m}^{-1}\right)$. Deformation of the solid boundaries at $\omega t=n \times 2 \pi$ is magnified. (For interpretation of the references to color in text, the reader is referred to the web version of the article.) 


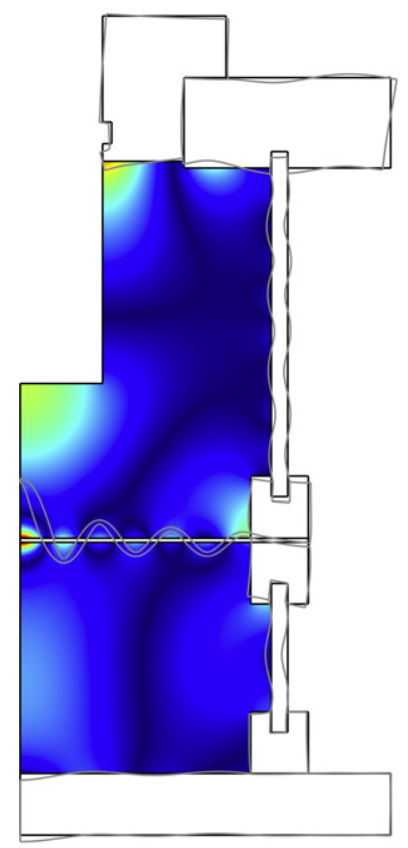

$$
\alpha=0 \mathrm{~m}^{-1}
$$

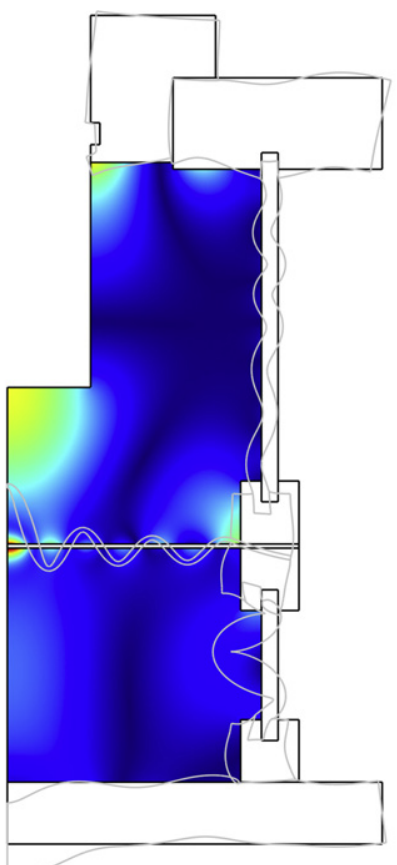

$\alpha=1 \mathrm{~m}^{-1}$

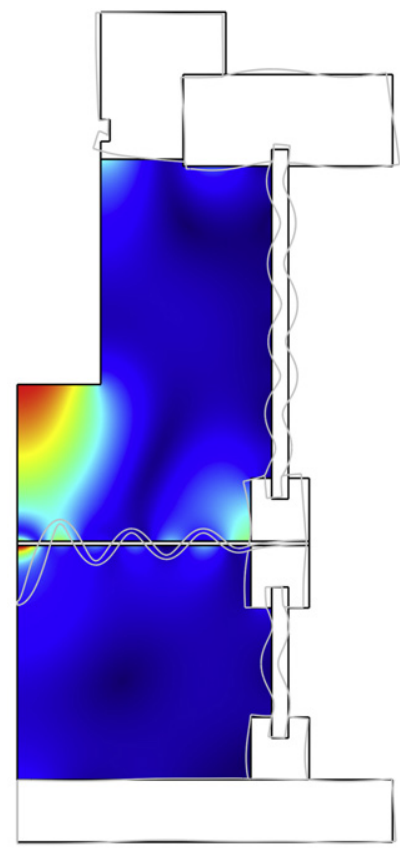

$\alpha=5 \mathrm{~m}^{-1}$

Fig. 8. Spatial distribution of the peak acoustic pressure $|P|$ in the reactor working in configuration $\lambda / 2-3 \lambda / 4$ near resonance peak IV for increasing attenuation coefficients. Dark blue regions represent pressure nodes while dark red regions correspond to pressure antinodes. The color-scales are different for each image. $|P|$ max $=3.84 \times 10^{3}$ bar $\left(\alpha=0 \mathrm{~m}^{-1}\right), 4.52 \times 10^{2} \operatorname{bar}\left(\alpha=1 \mathrm{~m}^{-1}\right)$ and $1.18 \times 10^{2} \operatorname{bar}\left(\alpha=5 \mathrm{~m}^{-1}\right)$. Deformation of the solid boundaries at $\omega t=n \times 2 \pi$ is magnified. (For interpretation of the references to color in text, the reader is referred to the web version of the article.)

esting, while configuration $3 \lambda / 4-\lambda / 2$ (highest transducer-electrode gap) would present the worst performance, from the stored acoustic energy point of view, working at any of its resonance peaks.

However, a high acoustic energy stored in the liquid does not necessary mean that cavitation takes place near the electrode. In order to check this consideration, the peak acoustic pressure on the electrode surface was also analyzed (Table 5 ). As it can be seen, the reactor with the shortest transducer-electrode gap (configuration $\lambda / 4-\lambda$ ) would reach the highest pressure amplitude working at peak $V$, followed by configuration $3 \lambda / 4-\lambda / 2$ at peak IV and configuration $\lambda / 2-3 \lambda / 4$ at peak $\mathrm{V}$. Therefore, the configuration with the shortest distance between the emitter and the electrode, working at peak $\mathrm{V}$ (Fig. 5), would be the most interesting from a sonoelectro-

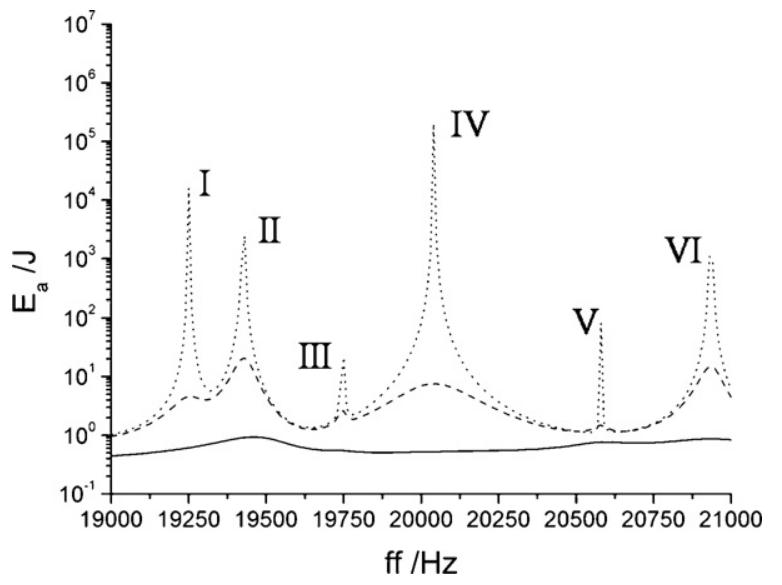

Fig. 9. Average acoustic energy stored in liquid in configuration $3 \lambda / 4-\lambda / 2$ for various attenuation coefficients: $\alpha=0 \mathrm{~m}^{-1}$ (dotted line), $\alpha=1 \mathrm{~m}^{-1}$ (dashed line) and $\alpha=5 \mathrm{~m}^{-1}$ (solid line) chemical point of view, as pressure antinodes alternating in both sides of the reactor reached higher pressure amplitudes in this case. Consequently, a higher amount of cavitating bubbles would form near the electrode, where mechanical phenomena such as acoustic streaming [25], shock waves [26] and others [27] would enhance mass-transport there $[28,29]$, also resulting in activation of the electrode surface [30]. The existence of a low-profile antinode at the bottom of the reactor would also provide a good location to place the counterelectrode (see file anim_025_100_peak5.gif included as Supplementary data). In addition, if cavitation-induced erosion of the transducer was also considered a key factor in order to chose a reactor design [31], configuration $3 \lambda / 4-\lambda / 2$ working at peak $V$ (Fig. 11) could be an interesting option from both sonoelectrochemical and emitter erosion points of view. As mentioned before, an antinode near the emitter would form for $\alpha=5 \mathrm{~m}^{-1}$, but its peak acoustic pressure would be significantly lower than the pressure amplitude near the electrode, which is located far enough of the emitter surface (see file anim_075_050_peak5.gif included as Supplementary data).

As a general discussion on the simulations, obtaining the optimum spatial distribution of the acoustic pressure accounting for the vibrations of the solid boundaries by setting a proper working frequency introduces a novel point of view in order to design efficient sonochemical/sonoelectrochemical reactors. Simulation studies generally neglect the deformations of the walls, where solid boundaries are treated as infinitely rigid $[20,32,33]$ or soft walls [21,34-36], or a combination of both [37], alleging different considerations in each case. Regarding these apparently controversial hypotheses, the present work shows that vibrations of the solid boundaries could deeply influence the system response in terms of both the spatial distribution of the acoustic pressure and the resonance frequencies of the system. Moreover, the consideration of solid boundaries as infinitely rigid or infinitely soft yields response curves which significantly differ from the ones obtained by properly 


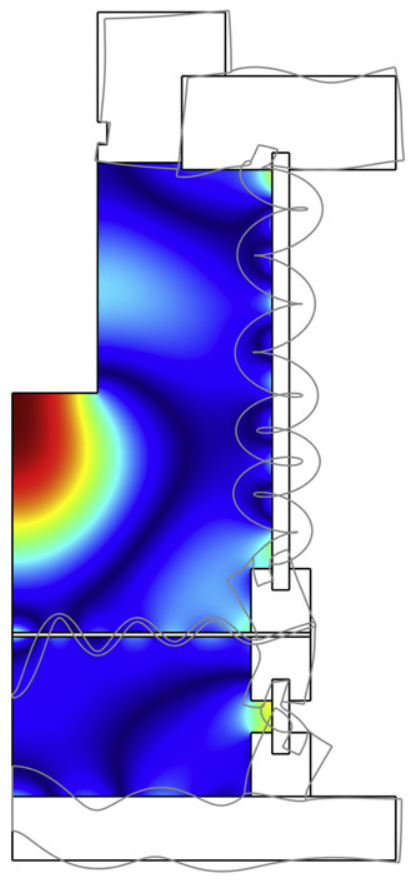

$$
\alpha=0 \mathrm{~m}^{-1}
$$

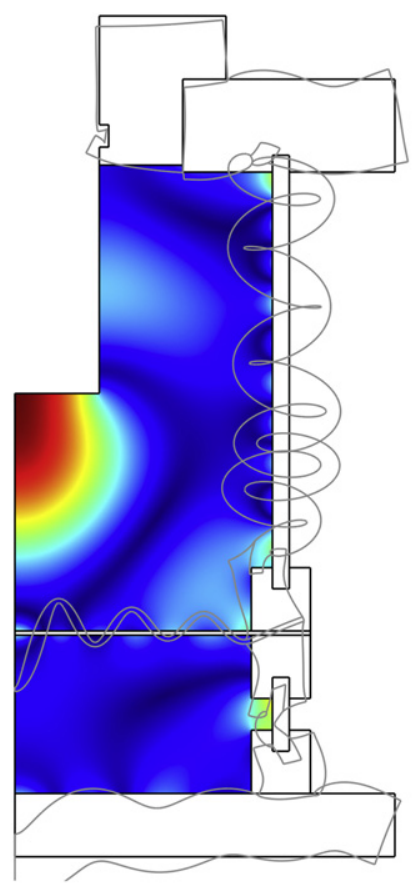

$\alpha=1 \mathrm{~m}^{-1}$

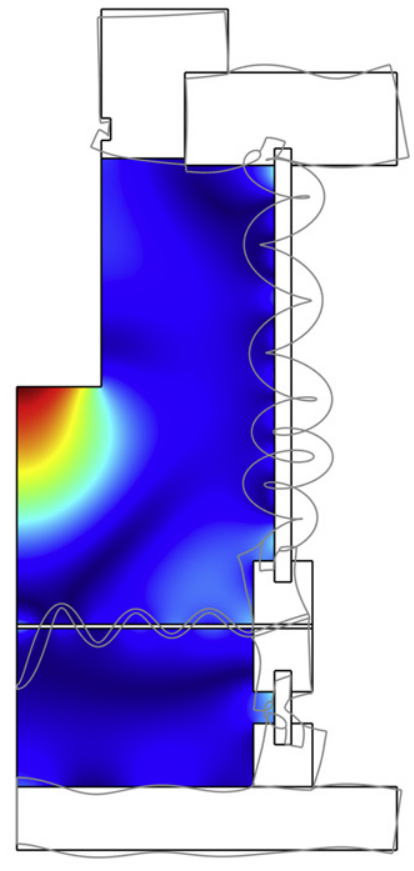

$\alpha=5 \mathrm{~m}^{-1}$

Fig. 10. Spatial distribution of the peak acoustic pressure $|P|$ in the reactor working in configuration $3 \lambda / 4-\lambda / 2$ near resonance peak II for increasing attenuation coefficients. Dark blue regions represent pressure nodes while dark red regions correspond to pressure antinodes. The color-scales are different for each image. $|P|$ max $=3.00 \times 10^{3}$ bar $\left(\alpha=0 \mathrm{~m}^{-1}\right), 2.84 \times 10^{2} \operatorname{bar}\left(\alpha=1 \mathrm{~m}^{-1}\right)$ and $8.12 \times 10$ bar $\left(\alpha=5 \mathrm{~m}^{-1}\right)$. Deformation of the solid boundaries at $\omega t=n \times 2 \pi$ is magnified. (For interpretation of the references to color in text, the reader is referred to the web version of the article.)

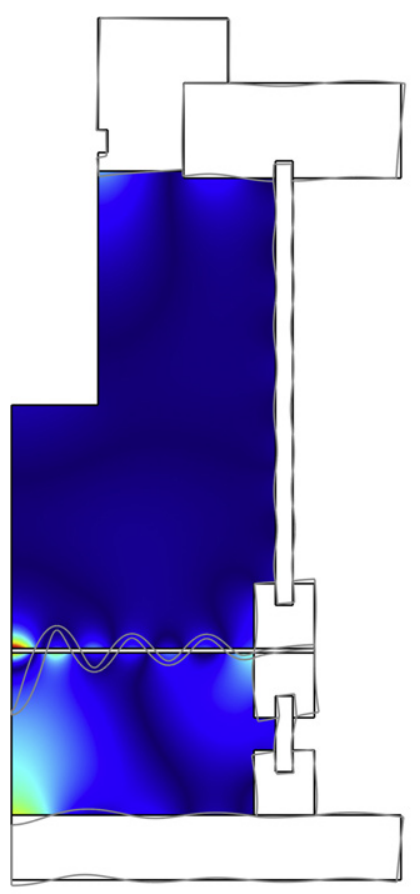

$$
\alpha=0 \mathrm{~m}^{-1}
$$

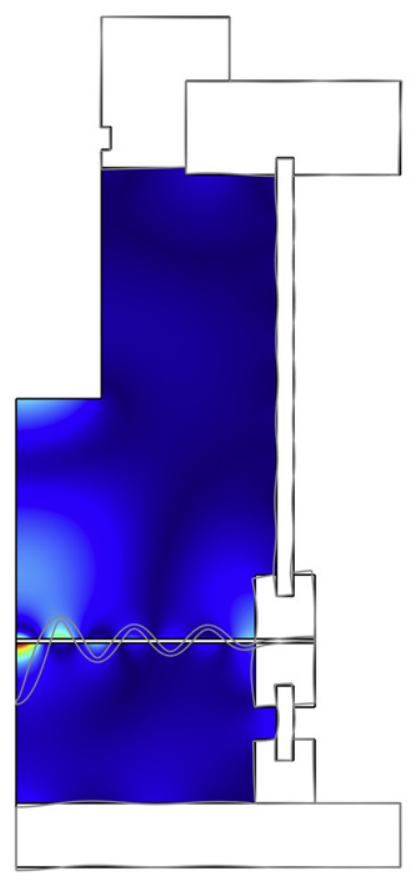

$\alpha=1 \mathrm{~m}^{-1}$

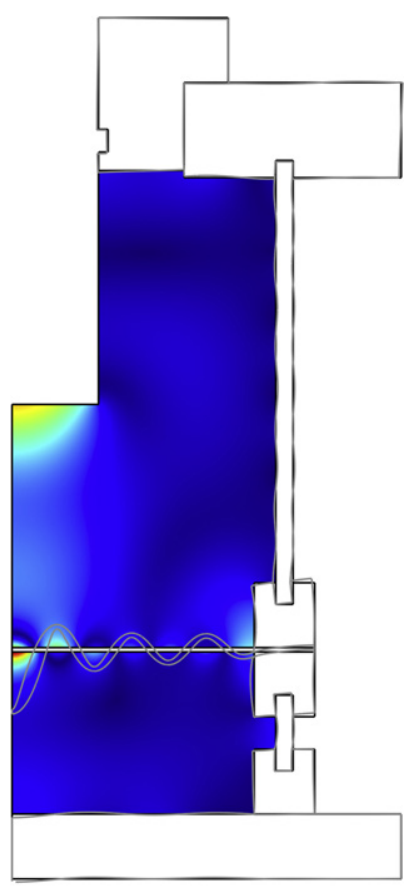

$$
\alpha=5 \mathrm{~m}^{-1}
$$

Fig. 11. Spatial distribution of the peak acoustic pressure $|P|$ in the reactor working in configuration $3 \lambda / 4-\lambda / 2$ near resonance peak $V$ for increasing attenuation coefficients. Dark blue regions represent pressure nodes while dark red regions correspond to pressure antinodes. The color-scales are different for each image. $|P|$ max $=1.25 \times 10^{3}$ bar $\left(\alpha=0 \mathrm{~m}^{-1}\right), 1.85 \times 10^{2}$ bar $\left(\alpha=1 \mathrm{~m}^{-1}\right)$ and $1.15 \times 10^{2}$ bar $\left(\alpha=5 \mathrm{~m}^{-1}\right)$. Deformation of the solid boundaries at $\omega t=n \times 2 \pi$ is magnified. (For interpretation of the references to color in text, the reader is referred to the web version of the article.) 


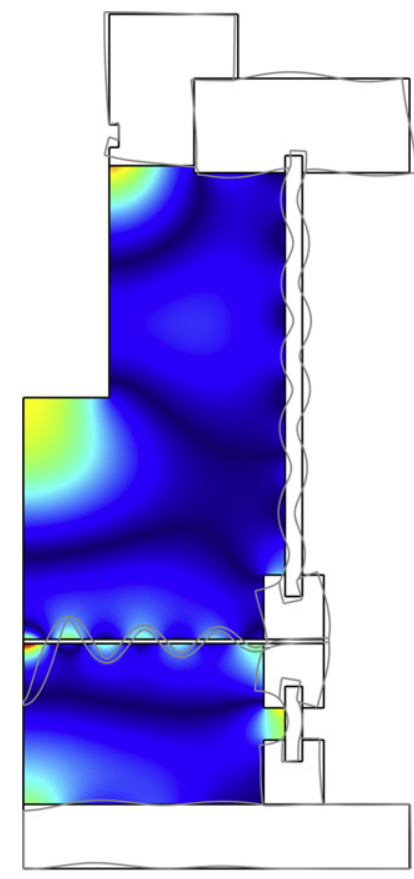

$\alpha=0 \mathrm{~m}^{-1}$

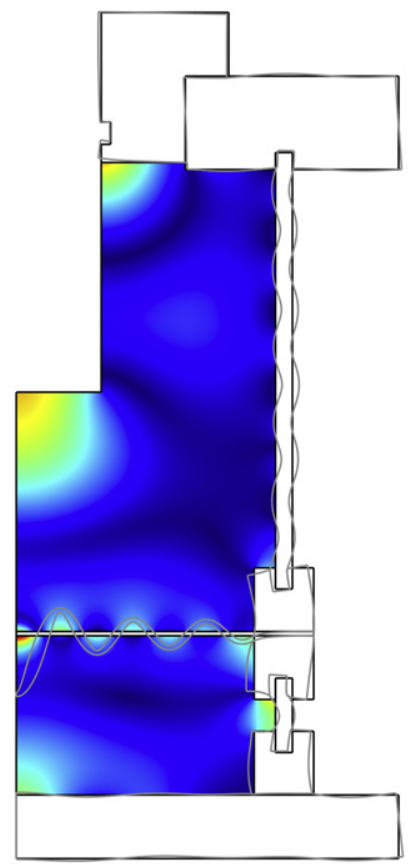

$\alpha=1 \mathrm{~m}^{-1}$

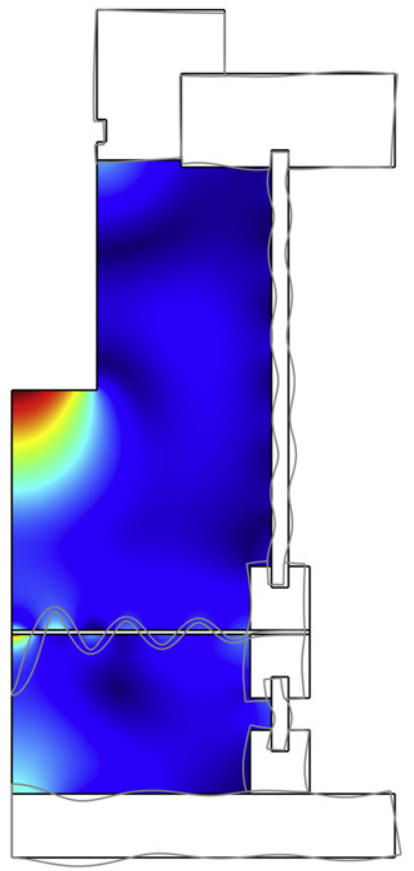

$\alpha=5 \mathrm{~m}^{-1}$

Fig. 12. Spatial distribution of the peak acoustic pressure $|P|$ in the reactor working in configuration $3 \lambda / 4-\lambda / 2$ near resonance peak VI for increasing attenuation coefficients. Dark blue regions represent pressure nodes while dark red regions correspond to pressure antinodes. The color-scales are different for each image. $|P|$ max $=2.77 \times 10^{3}$ bar $\left(\alpha=0 \mathrm{~m}^{-1}\right), 3.25 \times 10^{2}$ bar $\left(\alpha=1 \mathrm{~m}^{-1}\right)$ and $1.03 \times 10^{2}$ bar $\left(\alpha=5 \mathrm{~m}^{-1}\right)$. Deformation of the solid boundaries at $\omega t=n \times 2 \pi$ is magnified. (For interpretation of the references to color in text, the reader is referred to the web version of the article.)

taking into account the deformation of the solid [22]. An experimental evidence of the apparently suitable consideration of the solid boundaries as vibrating walls would be the diverse experimental works where the studied solution is not directly irradiated with an ultrasonic transducer (e.g. sonocatalytic degradation of dyes in low-frequency ultrasonic baths $[38,39]$ ).

Nevertheless, we must note that even though the main goal of the simulation of the acoustic field inside a sonochemical/sonoelectrochemical reactor is to locate pressure antinodes where cavitation events are expected, the maximum acoustic pressure amplitudes obtained in the simulations included in this work seemed unrealistic, especially when no attenuation was accounted for. This is due to two main reasons: (i) the assumed displacement of the transducer emitter surface (which was defined according to the supplier specifications) and (ii) the need of a more realistic model of attenuation by cavitating bubbles.

Regarding the first reason, some of the works found in the literature define the emitter as an "acoustic pressure source" where the pressure amplitude near the emitter is $P_{0}[20,21,32,35,36]$. In this sense, we tried to take a further step towards a more realistic approach to the matter, as we took the emitter surface displacement provided by the supplier and introduced it as a normal acceleration condition in the emitter boundary, assuming that the sonotrode behaves as an ideal acoustic piston. This displacement may not be exactly what the transducer actually experiences in any specific experimental conditions, but serve as an order of magnitude. In this sense, experimental measurements of the emitter surface displacement (i.e. laser vibrometry [40]) would help in order to obtain this parameter. Eventually, the complete simulation of the whole system including the ultrasonic transducer (i.e. piezoelectricity, pre-stress, etc.) should be carried out, in order to make a more rigorous approach to the problem. Related to this, some interesting works have been published in recent years where the piezoceramics, countermass and amplifier have been studied with FEM simulations [41-46]. Its inclusion in the model employed in this work would enhance the simulations, as vibrations of the horn tip could also be simulated in order to account for the lateral cavitation fields commonly observed during experiments.

Regarding the second reason, the lack of a definitive theory predicting the bubble spatial and size-distribution that would allow the correct calculation of the attenuation factor forced us to treat the latter quantity as a variable, but spatially uniform, parameter in the linear acoustics-based model employed in this work. This treatment allowed us to study how the spatial distribution of the acoustic pressure is affected by energy dissipation, pointing out the existence of an interesting travelling wave component in the propagation of the acoustic field from the emitter surface, even though the simulated geometry was a closed system where reflecting boundaries were defined and classic standing waves would be expected (see files anim_025_100_peak5.gif and anim_075_050_peak5.gif included as Supplementary data in order to visualize the travelling wave component). However, the correct value of a spatially non uniform attenuation factor should be strongly linked to the bubble repartition in the liquid, which in turn depends on the shape of the acoustic field [47]. Related to this, the long-term objective would be coupling both phenomena in our model to include the main physics of cavitation. This latter point is the most challenging task by far, and recent works by different authors involving non-linear acoustics are showing promising results [34,48-51]. Still, linear acoustics presents some intrinsic interest in the conception phase of sonochemi$\mathrm{cal} /$ sonoelectrochemical reactors, because although perturbed by the cavitation bubbles, linear resonance effects can still be visible. This is clearly supported by previous works by the authors [21], even though a rough model for solid boundary conditions was employed back then. It may be added that single bubble levitation cells, when used in non-degassed water, still exhibit a clear breathing standing wave with a bubble cloud wandering in the centre. 


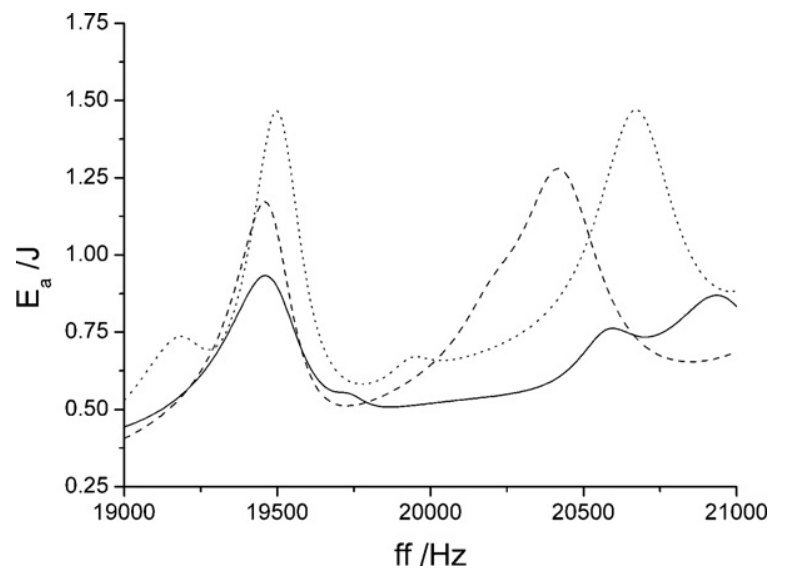

Fig. 13. Comparison of the average acoustic energy stored in liquid for $\alpha=5 \mathrm{~m}^{-1}$ : configuration $\lambda / 4-\lambda$ (dotted line), configuration $\lambda / 2-3 \lambda / 4$ (dashed line) and configuration $3 \lambda / 4-\lambda / 2$ (solid line).

Table 5

Dimensionless relative peak pressure amplitude near the electrode surface for each resonance peak of all the studied configurations. $|P|_{\max }=166.65$ bar reached near the electrode for configuration $\lambda / 4-\lambda$, peak $\mathrm{V}$.

\begin{tabular}{ll}
\hline Resonances & $|P| /|P|_{\max }$ \\
\hline$\lambda / 4-\lambda$-peak II & 0.40 \\
$\lambda / 4-\lambda$-peak V & 1.00 \\
$\lambda / 2-3 \lambda / 4$-peak I & 0.19 \\
$\lambda / 2-3 \lambda / 4$-peak IV & 0.71 \\
$3 \lambda / 4-\lambda / 2-$ peak II & 0.13 \\
$3 \lambda / 4-\lambda / 2$-peak V & 0.64 \\
$3 \lambda / 4-\lambda / 2$-peak VI & 0.46 \\
\hline
\end{tabular}

Therefore, linear acoustics, if properly used, remains as a useful tool.

\section{Conclusions}

In summary, this work shows how a basic variation of the transducer-electrode and electrode-wall gaps strongly modifies the sonoelectrochemical system response in terms of resonance peaks and structure of the acoustic field. Exciting the sonoelectrochemical system near a resonance frequency enables to reach large peak acoustic pressures and to locate pressure antinodes far from the transducer, in order to avoid cavitation-induced erosion of the emitter surface. However, this latter feature disappears for high attenuation coefficients, as its increase yields the formation and growth of a pressure antinode near the transducer surface. Even so, the election of a proper reactor configuration and working frequency enables to set high pressure antinodes near the electrode, which is very interesting from a sonoelectrochemical point of view. Related to the FEM simulations, the linear acoustics-based model accounting for the vibrations of the solid boundaries used in this work provides interesting results in order to design optimized sonoelectrochemical reactors, as it gives a qualitative idea of the acoustic field inside the reactors, and also provides the approximate location of the resonance frequencies of the system. Nevertheless, the theoretical nature of this work must be remarked, and further developments on the non-linear phenomena involved in the formation of cavitating bubbles and experimental validation are on its way.

\section{Acknowledgments}

I.T. and J.G.-G. thank the Generalidad Valenciana for its financial support under project ACOMP09/128 and grant FPA/2009/024.
I.T. also thanks the Spanish Education Ministry for his research scholarship. O.L. and J.G.-G. thank the Universidad de Alicante and Generalidad Valenciana for their support in scientific exchange missions. M.D.E. thanks Caja de Ahorros del Mediterraneo for her pre-doctoral grant.

\section{Appendix A. Supplementary data}

Animated files displaying the pressure field for configuration $\lambda / 2-3 \lambda / 4$ working at peak $V$ and configuration $3 \lambda / 4-\lambda / 2$ working at peak V.

\section{References}

[1] J. González-García, M.D. Esclapez, P. Bonete, Y. Vargas-Hernández, L. GaeteGarretón, V. Sáez, Current topics on sonoelectrochemistry, Ultrasonics 50 (2010) 318-322.

[2] V.O. Abramov, O.V. Abramov, A.E. Gekhman, V.M. Kuznetsov, G.J. Price, Ultrasonic intensification of ozone and electrochemical destruction of 1,3-dinitrobenzene and 2,4-dinitrotoluene, Ultrason. Sonochem. 13 (2006) 303-307.

[3] M.A. Oturan, I. Sirés, N. Oturan, S. Pérocheau, J.-L. Laborde, S. Trévin, Sonoelectro-Fenton process: a novel hybrid technique for the destruction of organic pollutants in water, J. Electroanal. Chem. 624 (2008) 329-332.

[4] H. Li, H. Lei, Q. Yu, Z. Li, X. Feng, B. Yang, Effect of low frequency ultrasonic irradiation on the sonoelectro-Fenton degradation of cationic red X-GRL, Chem. Eng. J. 160 (2010) 417-422.

[5] E. Joyce, T.J. Mason, S.S. Phull, J.P. Lorimer, The development and evaluation of electrolysis in conjunction with power ultrasound for the disinfection of bacterial suspension, Ultrason. Sonochem. 10 (2003) 231-234.

[6] J. González-García, Sonoelectrochemical synthesis of materials, in: M. Pankaj, Ashokkumar (Eds.), Theoretical and Experimental Sonochemistry Involving Inorganic Systems, Springer, Dordrecht/Heidelberg/London/New York, 2011.

[7] J. González-García, V. Sáez, J. Iniesta, V. Montiel, A. Aldaz, Electrodeposition of $\mathrm{PbO}_{2}$ on glassy carbon electrodes: influence of ultrasound power, Electrochem. Commun. 4 (2002) 370-373.

[8] V. Sáez, J. González-García, J. Iniesta, A. Frías-Ferrer, A. Aldaz, Electrodeposition of $\mathrm{PbO}_{2}$ on glassy carbon electrodes: influence of ultrasound frequency, Electrochem. Commun. 6 (2004) 757-761.

[9] V. Sáez, M.D. Esclapez, A.J. Frías-Ferrer, P. Bonete, I. Tudela, M.I. Díez-García, J. González-García, Lead dioxide film sonoelectrodeposition in acidic media: preparation and performance of stable practical anodes, Ultrason. Sonochem. (2010), doi:10.1016/j.ultsonch.2010.11.018.

[10] M.D. Esclapez, V. Sáez, D. Milán-Yáñez, I. Tudela, O. Louisnard, J. GonzálezGarcía, Sonoelectrochemical treatment of water polluted with trichloroacetic acid: from sonovoltammetry to pre-pilot plant scale, Ultrason. Sonochem. 17 (2010) 1010-1020.

[11] V. Sáez, M.D. Esclapez, I. Tudela, P. Bonete, O. Louisnard, J. González-García, $20 \mathrm{kHz}$ sonoelectrochemical degradation of perchloroethylene in sodium sulphate aqueous media: influence of the operational variables in batch mode, J. Hazard. Mater. 183 (2010) 648-654.

[12] V. Sáez, I. Tudela, M.D. Esclapez, P. Bonete, O. Louisnard, J. González-García, Sonoelectrochemical degradation of perchloroethylene in water: enhancement of the process by the absence of background electrolyte, Chem. Eng. J. (2011), doi:10.1016/j.cej.2011.01.052

[13] M.E. Abdelsalam, P.R. Birkin, A study investigating the sonoelectrochemical degradation of an organic compound employing Fenton's reagent, Phys. Chem. Chem. Phys. 4 (2002) 5340-5345.

[14] P.R. Gogate, V.S. Sutkar, A.B. Pandit, Sonochemical reactors: important design and scale up considerations with a special emphasis on heterogeneous systems, Chem. Eng. J. 166 (2011) 1066-1082.

[15] M. Ashokkumar, J. Lee, Y. Iida, K. Yasui, T. Kozuka, T. Tuziuti, A. Towata, The detection and control of stable and transient acoustic cavitation bubbles, Phys. Chem. Chem. Phys. 11 (2009) 10118-10121.

[16] S. Dähnke, F.J. Keil, Modeling of three-dimensional linear pressure fields in sonochemical reactors with homogeneous and inhomogeneous density distributions of cavitation bubbles, Ind. Eng. Chem. Res. 37 (1998) 848864.

[17] S. Dähnke, K.M. Swamy, F.J. Keil, Modeling of three-dimensional pressure fields in sonochemical reactors with an inhomogeneous density distribution of cavitation bubbles. Comparison of theoretical and experimental results, Ultrason. Sonochem. 6 (1999) 31-41.

[18] Y. Liu, T. Yamabuchi, T. Yoshizawa, S. Hirobayashi, Finite element simulation of coupled vibration modes in an ultrasonic cleaning tub: effect of the presence of a washing object, Acoust. Sci. Technol. 25 (2004) 173-176.

[19] K. Yasui, T. Kozuka, T. Tuziuti, A. Towata, Y. Iida, J. King, P. Macey, FEM calculation of an acoustic field in a sonochemical reactor, Ultrason. Sonochem. 14 (2007) 605-614.

[20] V.Sáez, A. Frías-Ferrer, J. Iniesta, J. González-García, A. Aldaz, E. Riera, Chacterization of a $20 \mathrm{kHz}$ sonoreactor. Part I: analysis of mechanical effects by classical and numerical methods, Ultrason. Sonochem. 12 (2005) 59-65. 
[21] J. Klíma, A. Frías-Ferrer, J. González-García, J. Ludvík, V. Sáez, J. Iniesta, Optimisation of $20 \mathrm{kHz}$ sonoreactor geometry on the basis of numerical simulation of local ultrasonic intensity and qualitative comparison with experimental results, Ultrason. Sonochem. 14 (2007) 19-28.

[22] O. Louisnard, J. Gonzalez-García, I. Tudela, J. Klíma, V. Sáez, Y. VargasHernández, FEM simulation of a sono-reactor accounting for vibrations of the boundaries, Ultrason. Sonochem. 16 (2009) 250-259.

[23] Hielscher Ultrasonics GmbH, UIP1000hd Operating Instructions, 2006.

[24] T.A. Davis, A column pre-ordering strategy for the unsymmetricpattern multifrontal method, ACM Trans. Math. Software 30 (2004) 165-195.

[25] J. Klíma, C. Bernard, Sonoassisted electrooxidative polymerisation of salicylic acid: role of acoustic streaming and microjetting, J. Electroanal. Chem. 462 (1999) 181-186.

[26] P.R. Birkin, D.G. Offin, P.F. Joseph, T.G. Leighton, Cavitation, shock waves and the invasive nature of sonoelectrochemistry, J. Phys. Chem. B 109 (2005) 16997-17005.

[27] J. Klíma, Application of ultrasound in electrochemistry. An overview of mechanisms and design of experimental arrangement, Ultrasonics 51 (2011) 202-209.

[28] E.L. Cooper, L.A. Coury Jr., Mass transport in sonovoltammetry with evidence of hydrodynamic modulation from ultrasound, J. Electrochem. Soc. 145 (1998) 1994-1999.

[29] D.J. Walton, S.S. Phull, A. Chyla, J.P. Lorimer, T.J. Mason, L. Burke, M. Murphy, R.G. Compton, J.C. Eklund, S.D. Page, Sonovoltammetry at platinum electrodes: surface phenomena and mass transport processes, J. Appl. Electrochem. 25 (1995) 1083-1090.

[30] R.G. Compton, J.C. Eklund, S.D. Page, G.H.W. Sanders, J. Booth, Voltammetry in the presence of ultrasound. Sonovoltammetry and surface effects, J. Phys. Chem. 98 (1994) 12410-12414.

[31] V.S. Sutkar, P.R. Gogate, Design aspects of sonochemical reactors: techniques for understanding cavitational activity distribution and effect of operating parameters, Chem. Eng. J. 155 (2009) 26-36.

[32] Z.-W. Shao, Q.-C. Le, J.-Z. Cui, Z.-Q. Zhang, Numerical simulation of standing waves for ultrasonic purification of magnesium alloy melt, Trans. Nonferrous Met. Soc. China 20 (2010) s382-s387.

[33] G. Memoli, P. Gélat, M. Hodnett, B. Zeqiri, The importance of temperature control in the operation of high power ultrasound reactors, in: 38th Annual Symposium of the Ultrasonic Industry Association (UIA), Vancouver, March 23rd-25th, 2009, article number 5404027.

[34] C. Vanhille, C. Campos-Pozuelo, Nonlinear ultrasonic standing waves: twodimensional simulations in bubbly liquids, Ultrason. Sonochem. 18 (2011) 679-682.

[35] V.S. Sutkar, P.R. Gogate, Theoretical prediction of cavitational activity distribution in sonochemical reactors, Chem. Eng. J. 158 (2010) 290-295.
[36] V.S. Sutkar, P.R. Gogate, L. Csoka, Mapping of cavitational activity in high frequency sonochemical reactor, Chem. Eng. J. 158 (2010) 296-304.

[37] S. Nomura, M. Nakagawa, Analysis of an ultrasonic field attenuated by oscillating cavitation bubbles, Acoust. Sci. Technol. 22 (2001) 283-291.

[38] N. Shimizu, C. Ogino, M.F. Dadjour, T. Murata, Sonocatalytic degradation of methylene blue with $\mathrm{TiO}_{2}$ pellets in water, Ultrason. Sonochem. 14 (2007) 184-190.

[39] Y.L. Pang, A.Z. Abdullah, S. Bhatia, Optimization of sonocatalytic degradation of Rhodamine $\mathrm{B}$ in aqueous solution in the presence of $\mathrm{TiO}_{2}$ nanotubes using response surface methodology, Chem. Eng. J. 166 (2011) 873-880.

[40] L. Zipser, H. Franke, Laser-scanning vibrometry for ultrasonic transducer development, Sens. Actuator A Phys. 110 (2004) 264-268.

[41] A. Iula, F. Vazquez, M. Pappalardo, J.A. Gallego, Finite element threedimensional analysis of the vibrational behaviour of the Langevin-type transducer, Ultrasonics 40 (2002) 513-517.

[42] A. Iula, L. Parenti, F. Fabrizi, M. Pappalardo, A high displacement ultrasonic actuator based on a flexural mechanical amplifier, Sens. Actuator A Phys. 125 (2006) 118-123.

[43] J.A. Gallego-Juárez, E. Riera, V.M. Acosta-Aparicio, Modal interactions in highpower ultrasonic processing transducers, AIP Conf. Proc. 1022 (2008) 595-604.

[44] A. Cardoni, E. Riera, A. Blanco-Blanco, J. Gallego-Juarez, V. Acosta-Aparicio, On the nonlinear dynamics of plate-transducers, in: 2009 IEEE International Ultrasonics Symposium (UIS) Proceedings, Rome, September 20th-23rd, 2009, pp. 2576-2579.

[45] J.A. Gallego-Juárez, G. Rodriguez, V. Acosta, E. Riera, Power ultrasonic transducers with extensive radiators for industrial processing, Ultrason. Sonochem. 17 (2010) 953-964.

[46] A. Abdullah, A. Pak, Correct prediction of the vibration behavior of a high power ultrasonic transducer by FEM simulation, Int. J. Adv. Manuf. Technol. 39 (2008) 21-28.

[47] R. Mettin, P. Koch, W. Lauterborn, D. Krefting, Modeling acoustic cavitation with bubble redistribution, in: Sixth International Symposium on Cavitation-CAV2006 (paper 75), Wageningen, The Netherlands, 2006, pp. 125-129.

[48] B. Dubus, C. Vanhille, C. Campos-Pozuelo, C. Granger, On the physical origin of conical bubble structure under an ultrasonic horn, Ultrason. Sonochem. 17 (2010) 810-818

[49] O. Louisnard, Nonlinear attenuation of sound waves by inertial cavitation bubbles, Phys. Procedia 3 (2010) 735-742.

[50] O. Louisnard, A simple model of ultrasound propagation in a cavitating solid. Part I: Theory, nonlinear attenuation and traveling wave generation, Ultrason. Sonochem. (2011), in press.

[51] O. Louisnard, A simple model of ultrasound propagation in a cavitating solid. Part II: Primary Bjerknes force and bubble structures, Ultrason. Sonochem. (2011), in press. 